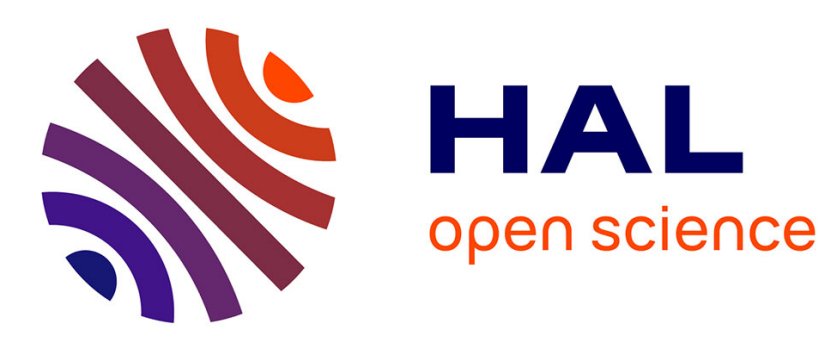

\title{
Study on the temperature dependence of the bulk modulus of polyisoprene by molecular dynamics simulations
}

\author{
Julie Diani, Bruno Fayolle, Pierre Gilormini
}

\section{- To cite this version:}

Julie Diani, Bruno Fayolle, Pierre Gilormini. Study on the temperature dependence of the bulk modulus of polyisoprene by molecular dynamics simulations. Molecular Simulation, 2008, 34 (10-15), pp.1143-1148. 10.1080/08927020801993388 . hal-00515030

\author{
HAL Id: hal-00515030 \\ https://hal.science/hal-00515030
}

Submitted on 4 Sep 2010

HAL is a multi-disciplinary open access archive for the deposit and dissemination of scientific research documents, whether they are published or not. The documents may come from teaching and research institutions in France or abroad, or from public or private research centers.
L'archive ouverte pluridisciplinaire HAL, est destinée au dépôt et à la diffusion de documents scientifiques de niveau recherche, publiés ou non, émanant des établissements d'enseignement et de recherche français ou étrangers, des laboratoires publics ou privés. 


\section{Molecular Simulation}

Journal of

Experimental Nanoscience

Taylor \& Francis

Taylor \& Francis Group

\section{Study on the temperature dependence of the bulk modulus of polyisoprene by molecular dynamics simulations}

\begin{tabular}{|r|l|}
\hline Journal: & Molecular Simulation/Journal of Experimental Nanoscience \\
\hline Manuscript ID: & GMOS-2008-0006.R1 \\
\hline Journal: & Molecular Simulation \\
\hline Date Submitted by the \\
Author: & 08-Feb-2008 \\
\hline Complete List of Authors: & $\begin{array}{l}\text { Diani, Julie; LIM, ENSAM Paris } \\
\text { Fayolle, Bruno; LIM } \\
\text { Gilormini, Pierre; LIM }\end{array}$ \\
\hline Keywords: & $\begin{array}{l}\text { Molecular dynamics simulations, polyisoprene, bulk modulus, glass } \\
\text { transition, compressibility }\end{array}$ \\
\hline
\end{tabular}

\section{SCHOLARONE"}

Manuscripts 


\title{
Study on the temperature dependence of the bulk modulus of polyisoprene by molecular dynamics simulations
}

\author{
J. DIANI", B. FAYOLLE and P. GILORMINI \\ Laboratoire d'Ingénierie des Matériaux, CNRS, ENSAM Paris, 151 bd de l'Hôpital, 75013 Paris, France.
}

The temperature dependence of the bulk modulus of polyisoprene has been studied using molecular dynamics simulations. Virtual polyisoprenes have been submitted to volume contractions above and below the glass transition. Bulk modulus has been observed to be linearly dependent on temperature both above and below the glass transition respectively, and it dropped by a factor of about 2 while temperatures was risen above the glass transition. By monitoring the energy changes during volume contractions, it was observed that the bulk modulus arises mainly from the Van der Waals interactions. Nevertheless, the entropy contribution to the bulk modulus becomes significant above the glass transition. At a first order, the entropy part of the bulk modulus can be considered as independent on the temperature.

Keywords: Molecular dynamics simulations; Polyisoprene; Bulk modulus; Glass transition; Compressibility.

\section{Introduction}

The small-strain elastic mechanical behaviour of isotropic materials is fully characterized by two parameters. One can choose for instance, the Young's modulus and the bulk modulus. Dealing with amorphous polymers, a specific dependence of these two parameters vs. temperature is observed. The Young's modulus is known to drop by one to three orders of magnitude while temperature rises above the glass transition temperature [1]. The bulk modulus is known to decrease by a factor of only 2 or 3 while temperature rises above the glass transition temperature [2]. The temperature dependence of the Young's modulus has been explained well in the literature [3]: above the glass transition, molecular mobility increases and enables entropic elasticity. However, the moderate drop of the bulk modulus is still not fully understood and the question of a possible entropy contribution to the bulk deformation is still open [4]. Tabor's [5] theoretical study of bulk modulus of rubber suggests that it arises from the Van der Waals interactions and that it is not related to the tensile modulus. Also, Theodorou and Suter's atomistic modelling [6] shows that entropy contributions to the elastic response to deformation can be

*Corresponding author: fax: (33) 144246290.

Email: julie.diani@paris.ensam.fr 
neglected in polymer glasses. Conversely, Matheson's [4,7] reading of various experimental data of the literature seems to indicate that the entropy contribution cannot be generally neglected when considering the compressibility of polymeric glasses and rubbers. In this paper, we propose to address the question of the physical source of the bulk modulus of rubber (polyisoprene cis1-4) using molecular dynamics simulations.

Recently, the access to reliable affordable computers has contributed to the development of molecular dynamics simulations, and fully atomistic studies providing physical understandings on the mechanical behaviour of polymers are now possible. A number of studies have shown the ability of molecular dynamics to successfully reproduce the glass transition for amorphous polymers [8-11]. It is then reasonable to consider MD simulations to provide physical insight into the bulk modulus. This requires applying hydrostatic loadings, which are favourable for easy and fast MD simulations [12]. In such loadings, the number of degrees of freedom is reduced in comparison to uniaxial tension or shear. Using Materials Studio software [13], we built several virtual polyisoprenes and we applied hydrostatic compressions to the materials at various temperatures. During the simulations, the cell volume, pressure, and temperature were monitored, as well as the bonded, nonbond and kinetic energies. These data were used in a classical thermodynamics analysis to assess the physical source of the bulk modulus below and above the glass transition temperature.

\section{General theory}

We limit ourselves to hydrostatic loadings, which can result from an applied hydrostatic pressure $p$ or from a volume contraction. The first law of thermodynamics relates a small change in internal energy $U$ to small changes in entropy $S$ and volume $V$, during quasi-static processes:

$$
d U=T d S-p d V .
$$

Considering the volume temperature $T$ as two independent variables, Equation (1) leads to:

$$
\left.\frac{\partial U}{\partial T}\right|_{V}=\left.T \frac{\partial S}{\partial T}\right|_{V} \text { and }\left.\frac{\partial U}{\partial V}\right|_{T}=-p+\left.T \frac{\partial S}{\partial V}\right|_{T} .
$$

The isothermal bulk modulus is defined as:

$$
B(T, V)=-V\left(\frac{\partial p}{\partial V}\right)_{T},
$$

and, by substituting Equation (2) in Equation (3) one gets

$$
B(T, V)=\left.V \frac{\partial^{2} U}{\partial V^{2}}\right|_{T}-\left.V T \frac{\partial^{2} S}{\partial V^{2}}\right|_{T}
$$

which allows to define the internal energy contribution and the entropy contribution to the bulk modulus as:

$$
B^{U}(T, V)=\left.V \frac{\partial^{2} U}{\partial V^{2}}\right|_{T} \text { and } B^{S}(T, V)=-\left.V T \frac{\partial^{2} S}{\partial V^{2}}\right|_{T}
$$

In small strain elasticity, the bulk modulus refers to moderate volume contractions and is defined as 
$B\left(T, V_{0}(T)\right)=B_{0}(T)$, where $V_{0}(T)$ corresponds to the volume of the material at temperature $T$ and at atmospheric pressure. For various temperatures, we applied moderate volume contractions and used Equation (3) to obtain the bulk modulus as a function of temperature. During MD simulations, the internal energy of the system is computed as the sum of the bonded energy, the nonbond energy and the kinetic energy:

$$
U=E_{B}+E_{N B}+K_{c}
$$

The nonbond energy consists of the Van der Waals energy, the electrostatic energy and the hydrogen bond terms, while the bonded energy accounts for the valence interactions, such as bond stretching, dihedral angle torsion, valence angle bending, inversion.... The force-field used in Material Studio is COMPASS [14], which is an abinitio-based force-field that has been validated on isolated molecules [15,16]. In COMPASS, the Van der Waals interactions are defined by a 9-6 Lennard-Jones potential. Having access to the variation of the internal ernergy $U$ as a function of $V$, we can assess the internal energy contribution $B^{U}\left(T, V_{0}(T)\right)$ through Equation (5) and, finally, the entropy contributions to $B_{0}(T)$ is deduced.

\section{Molecular dynamics simulations}

\subsection{Procedure}

Fully atomistic simulations were performed on periodic systems of cis-polyisoprene. The virtual materials were built using an amorphous cell generator provided in Materials Studio, with periodic boundary conditions. Each parent cell contains 5 chains of 300 monomers. The cubic parent cells were built at a high temperature (above the expected glass transition) of $400 \mathrm{~K}$ to ensure a better stability. Each system was then submitted to consecutive, energy minimization, 1 ns-NVT MD simulation (number of atoms, volume and temperature remain constant in a NVT ensemble), and 1 ns-NPT MD simulations (number of atoms, pressure and temperature remain constant in a NPT ensemble). This provided reasonably stable amorphous structures at atmospheric pressure and $400 \mathrm{~K}$. The structures were then cooled down to $100 \mathrm{~K}$ with a series of $25 \mathrm{~K}$ to $50 \mathrm{~K}$ jumps with intermediate NPT MD steps running for 500 ps with a time step of $1 \mathrm{fs}$. The duration of the simulations is constrained by the relatively high number of atoms (19 510) and by the fact that we use fully atomistic simulations (and not coarse grain simulations). Nevertheless, to ensure that 500 ps were long-enough duration, we monitored the cell specific volume during $1 \mathrm{~ns}$ for NPTs at a low and a high temperature. As shown in Figure 1, the volume has converged before $500 \mathrm{ps}$.

Six cells were used to calculate the glass transition of the virtual materials. From these six cells, two were submitted to volume contractions in order to study the Bulk modulus temperature dependence. For each temperature, both cells were submitted to moderate $1.5 \%$ and $3 \%$-volume contractions in order to calculate $B_{0}(T)$ according to Equation (3). Cell 1 simulations were run using Berendsen [17] barostat and thermostat, while cell 2 simulations used the Andersen [18] barostat and thermostat. Both methods provided similar results. 
In addition, larger volume contractions were applied to both cells in order to better analyze the energy variations.

\subsection{Results}

During 1 bar-isobaric cooling, we observe a linear decrease of the specific volume at both high and low temperatures with decreasing temperature. The logarithm of the specific volume of the cells (volume per unit mass) is plotted vs. the temperature in Figure 2, so that the slope gives the coefficient of volumic thermal expansion directly. The change in the thermal contraction defines a clean glass transition at $\mathrm{T}_{\mathrm{g}} \sim 250 \mathrm{~K}$, which is $50 \mathrm{~K}$ above experimental values reported in the literature ( 203 K at $10 \mathrm{~K} / \mathrm{min})$ [19] but is in good accordance with former MD studies on cis-polyisoprene [11]. From Figure 2, the expansion coefficients above and below $\mathrm{T}_{\mathrm{g}}$ can be evaluated as $\alpha_{r}=5.15 \times 10^{-4} \mathrm{~K}^{-1}$ and $\alpha_{g}=2.97 \times 10^{-4} \mathrm{~K}^{-1}$, respectively, in qualitatively good agreement with values reported in the literature [19], $\alpha_{r}=6.16 \times 10^{-4} \mathrm{~K}^{-1}$ and $\alpha_{g}=2.07 \times 10^{-4} \mathrm{~K}^{-1}$.

In order to asses the material compressibility, we first submitted cell 1 to an increasing volume contraction at $350 \mathrm{~K}$. The resulting pressure is plotted in Figure 3 as a function of the logarithm of the specific volume of the cell. We note that the material stiffens as the volume decreases, which indicates that compressibility decreases with volume contraction. This result is consistent with rubber behaviour reported in the literature [20]. As previously mentioned, the bulk modulus is related to the material response for small volume contractions and is defined by the linear approximation of $p$ vs. $\ln (V(T))$ according to Equation (3). Figure 3 illustrates how $B_{0}(350)$ is estimated to be $1460 \mathrm{MPa}$. For each temperature, the bulk modulus $B_{0}(T)$ is calculated using three states: atmospheric pressure and resulting volume $V_{0}(T), 1.5 \%$-volume contraction, $3 \%$-volume contraction. In Figure 4, the values of the bulk modulus are plotted vs. temperature for cells 1 and 2, and a linear relation can be considered both above and below the glass transition temperature. Below $T_{g}$, a rise in temperature produces a decrease of the bulk modulus twice as important as above $T_{g}$. We obtained a value of $2100 \mathrm{MPa}$ for $B_{0}(300)$, which is in good agreement with the value of $2020 \mathrm{MPa}$ reported in the literature [21]. Around the glass transition, $B_{0}(T)$ varies more significantly, and the ratio of the bulk modulus at $200 \mathrm{~K}\left(50 \mathrm{~K}\right.$ below $\left.T_{g}\right)$ over the bulk modulus at $300 \mathrm{~K}\left(50 \mathrm{~K}\right.$ above $\left.T_{g}\right)$ is close to 2 . A change of such a magnitude through the glass transition is consistent with the experimental behaviour reported in amorphous polymers [2]. Therefore, the bulk modulus temperature dependence presented by our virtual materials is representative of the behaviour of a real polyisoprene. Consequently, in an attempt to provide some physical understandings of the material compressibility, the system energy changes have been monitored during volume contractions at fixed temperature and during temperature changes at fixed volume.

Cell 1 was submitted to several volume contractions at three fixed temperatures $350 \mathrm{~K}, 250 \mathrm{~K}$ and $150 \mathrm{~K}$ and stabilized by NVT simulations. During these simulations, we have collected the changes of the three component 
of the internal energy (Equation (6)): the bonded energy, the nonbonded energy and the kinetic energy. Considering the latter, only a change of temperature affects $K_{c}$ since by definition $K_{c}=3 N k_{B} T / 2$, where $k_{B}$ is the Boltzmann's constant, $N$ is the number of atoms and $T$ the temperature. Consequently, the kinetic energy remains constant during volume contractions at constant temperature. In Figure 5, the bonded energy is plotted vs. the cell specific volume for the three temperatures. In order to provide a simple read of the data in a single Figure for all temperatures, the bonded energy was normalized for each temperature by its maximum value, which was obtained for the highest contraction (or equivalently smallest volume). At $350 \mathrm{~K}$, above $T_{g}$, the bonded energy remains constant through volume contractions. At $150 \mathrm{~K}$, the bonded energy fluctuates around a plateau value for moderate contractions, including those applied to determine the bulk modulus. Then, for severe volume contractions, the bonded energy increases drastically. At $250 \mathrm{~K}$, the same trend for the bonded energy as at $150 \mathrm{~K}$ is recognized, though it is subtler: the bonded energy does not vary for moderate volume contractions and it increases evidently for high contractions. Therefore, it can be concluded that, at both high and low temperatures, the bonded energy does not vary significantly when volume contraction keeps within the range used to compute the bulk modulus.

As mentioned previously, three sources may contribute to the nonbonded energy: the Van der Waals interactions, the electrostatic forces and the hydrogen bonds. Since polyisoprene has no hydrogen bond and electrostatic forces are found to remain constant with temperature and volume contractions, the computed nonbond energy changes amount to Van der Waals energy changes only. The latter are shown in Figure 6 vs. the cell specific volume. For each temperature, the curve shape is reminiscent of a Lennard-Jones potential. The Van der Waals energy per unit mass varies with temperature, but Figure 7 shows that the three curves coincide reasonably if a suitable vertical translation is applied. This result allows the Van der Waals energy per unit mass to be written as:

$$
E_{V d W}(T, V)=E_{V d W}\left(T_{0}, V\right)+\beta\left(T-T_{0}\right),
$$

with $\beta=356 \mathrm{~J} \cdot \mathrm{kg}^{-1} \cdot \mathrm{K}^{-1}$ if $T_{0}$ is taken as $350 \mathrm{~K}$. From Figures 5 and 6 , we conclude that when the virtual polyisoprenes are submitted to moderate volume contractions (which are the contractions that define the bulk modulus), only the Van der Waals energy varies significantly among the various terms contributing to the internal energy. Cell 2 was submitted to volume contractions at $400 \mathrm{~K}, 350 \mathrm{~K}$ and $150 \mathrm{~K}$ and lead to similar results than those presented above; they will be used to strengthen the analysis in the next section. The data obtained from the volume contractions at constant temperature will be useful to determine $B^{U}\left(T, V_{0}(T)\right)$ and, by subtracting to $B_{0}(T)$, to estimate the contribution of entropy to the bulk modulus.

\section{Van der Waals contribution to the bulk modulus}

Since the Van der Waals energy changes, in the range of volume contractions considered to compute the bulk modulus, leads the internal energy changes, Equation (5) gives 


$$
B_{0}^{U}(T)=B^{U}\left(T, V_{0}(T)\right)=\left.\left.V_{0}(T) \frac{\partial^{2} U}{\partial V^{2}}\right|_{T} \approx V_{0}(T) \frac{\partial^{2} E_{V d W}}{\partial V^{2}}\right|_{T} .
$$

Figure 7 suggests that a simple equation should be able to approach the Van der Waals energy per unit mass. Actually, the 9-6 Lennard-Jones potential used at the local scale to define the Van der Waals interactions suggests trying to apply the same function to the overall Van der Waals energy of the cell, and the Equation (7) would write:

$$
E_{V d W}(T, V)=\frac{a}{V^{3}}-\frac{c}{V^{2}}+\beta\left(T-T_{0}\right) .
$$

As shown Figure 8, Equation (9) does fit the Van der Walls energies for cells 1 and 2 relatively well when $a=1.90$ $10^{-3} \mathrm{~J} . \mathrm{m}^{9} . \mathrm{kg}^{-4}, c=2.81 \mathrm{~J} \cdot \mathrm{m}^{6} \cdot \mathrm{kg}^{-3}$ are used for cell 1 and $a=2.010^{-3} \mathrm{~J} \cdot \mathrm{m}^{9} \cdot \mathrm{kg}^{-4} c=2.96 \mathrm{~J}^{-\mathrm{m}^{6}} \cdot \mathrm{kg}^{-3}$ for cell2.

By substitution of Equation (9) in Equation (8), the following simple form is obtained for $B_{0}^{U}(T)$ :

$$
B_{0}^{U}(T)=\frac{12 a}{V_{0}^{4}(T)}-\frac{6 c}{V_{0}^{3}(T)}
$$

For any temperature $T, V_{0}(T)$ can be evaluated from the two-part linear fit shown in Figure 2, and thus $B_{0}^{U}(T)$ can be computed from Equation (10) and compared to $B_{0}(T)$. This comparison is presented in Figure 9 for cells 1 and 2. For temperatures below the glass transition, it can be observed that the bulk modulus $B_{0}(T)$ is close to $B_{0}^{U}(T)$, though the values of $B_{0}(T)$ are slightly underestimated in cell 2. Since the latter is computed from the Van der Waals energy only, which dominates the internal energy, no significant entropy contribution is to be noticed in the glassy state. In contrast, $B_{0}^{U}(T)$ overestimates the bulk modulus above the glass transition. This overestimation indicates that entropy does contribute to the bulk modulus above the glass transition. We note that the entropy affects the bulk modulus by reducing it (in the sense that $B_{0}(T)$ is lower than $B_{0}^{U}(T)$ ). In Figure 9 , plotting the parallel to the values of $B_{0}^{U}(T)$ passing through the values of $B_{0}(T)$ shows that the entropy contribution $B_{0}^{S}(T)$ to $B_{0}(T)$ may be considered as independent of temperature to the first order, with an average value of $-400 \mathrm{MPa}$ for cell 1 and $-300 \mathrm{MPa}$ for cell 2.

\section{Conclusion}

Molecular dynamics simulations were performed on virtual polyisoprenes in order to investigate the physical source of the bulk modulus above and below the glass transition. The materials were submitted to moderate volume contractions at fixed temperature through NVT simulations, in order to estimate the bulk modulus dependence on temperature. The bulk modulus values thus obtained were shown to be in qualitative and quantitative good agreements with data reported in the literature. In particular, the bulk modulus showed a drop by a factor of about 2 at the glass transition. Investigating the internal energy change during volume contractions 
at constant temperature, we observed that for moderate contractions, sufficient to estimate the bulk modulus, the internal energy change arises from the Van der Waals energy only. Hence, by approaching the overall Van der Waals energy by a 9-6 Lennard-Jones potential, and by applying a classical thermodynamic analysis, the internal energy contribution to the bulk modulus was estimated below and above the glass transition. This contribution was shown to reproduce fairly well the bulk modulus below the glass transition but to overestimate it above. In the latter temperature range, entropy changes lessen the bulk modulus by an amount that can be considered as temperature independent to first order.

\section{Acknowledgments}

This work was supported by grant JC05_43403 from the French Agence Nationale de la Recherche.

\section{References}

[1] F. Bueche, Physical properties of polymers, Interscience NY, (1962).

[2] J. Bicerano, Predictions of polymer properties, $3^{\text {rd }}$ ed., Marcel Dekker Inc.: NY, (2002), p. 398.

[3] L.R.G. Treloar, Physics of rubber elasticity, $3^{\text {rd }}$ ed., Oxford University press, (1975).

[4] R.R. Matheson, Significance of entropic factor in mechanical deformation of polymeric glasses, Macromolecules 20 (1987), pp. 1847-1851.

[5] D. Tabor, The bulk modulus of rubber, ,Polymer 35 (1994), pp. 2759-2763.

[6] D.N. Theodorou, U.W. Suter, Atomistic modeling of mechanical properties of polymeric glasses, Macromolecules 19 (1986), pp. 139-154.

[7] R.R. Matheson, Measurements of entropic factors in the mechanical deformation of polycarbonate and natural rubber, Macromolecules 20 (1987), pp.1851-1855.

[8] D. Rigby, R.J. Roe, Molecular dynamics simulations of polymer liquid and glass. I. glass transition, J. Chem. Phys. 87 (1987), pp. 7285-7292.

[9] J. Han, R.H. Gee, R.H. Boyd, Glass-transition temperatures of polymers from molecular-dynamics simulations, Macromolecules 27 (1994),pp. 7781-7784.

[10] L. Yang, D.J. Srolobitz, A.F. Yee, Molecular dynamics study of isobaric and isochoric glass transitions in a model amorphous polymer, J. Chem. Phys. 110(1999), 7058-7069.

[11] M. Fukuda, H. Kikuchi, Chain dynamics and conformation transition of cis-polyisoprene: Comparison between melt and subglasses state by molecular dynamics simulation, J. chem. Phys. 113 (2000), pp. 4433-4444. 
[12] S.B. Sane, T. Cagin, W.A. Goddard, W.G. Kauss, Molecular dynamics simulations to compute the bulk response of amorphous PMMA, J. comp. Aid. Mater. Des. 8 (2002), pp. 87-106.

[13] Accelrys Inc. MATERIALS STUDIO. 2003, (Accelrys Inc., San Diego, CA.)

[14] H. Sun, COMPASS: An ab-initio force-field optimized for condensed-phase applications-Overview oith details on alkane and benzene compounds, J. Phys. Chem. 102 (1998), pp. 7338-7364.

[15] J. Yang, Y. Ren, A.M. Tian, H. Sun, COMPASS force field for 14 inorganic molecules, $\mathrm{He}, \mathrm{Ne}, \mathrm{Ar}, \mathrm{Kr}, \mathrm{Xe}$, H-2, O-2, N-2, NO, CO, CO2, NO2, CS2, and SO2, in liquid phase, J. Phys. Chem. B. 104 (2000), pp. 4951-4957.

[16] S.W. Bunte, H. Sun, Molecular modeling of energetic materials: the parameterization and validation of nitrate esters in the COMPASS force field, J. Phys. Chem. B. 104 (2000), pp. 2477-2489.

[17] H.J.C. Berendsen, J.P.M. Postma, W.F. Van Gunsteren, A. DiNola, J.R. Haak, Molecular-dynamics with coupling to an external bath, J. Chem. Phys. 81 (1984), pp. 3684-3690.

[18] H.C. Andersen, Molecular dynamics simulations at constant pressure and/or temperature, J. Chem. Phys. 72 (1980), pp. 2384-2393.

[19] A.V. Tobolski, J.E. Mark, Polymer Science and Materials, Krieger, NY, 1980.

[20] L.H. Adams, R.E. Gibson, The compressibility of rubber, J. Washington. Acad. Sci. 20 (1930), pp. 213-223.

[21] B.P. Holownia, Effect of carbon black on Poisson's ratio of elastomers, J. Instit. Rubber Ind., 8 (1974), pp. 157-164.

Figure 1. Convergence of the specific volume of the virtual material vs. simulation duration during NPT ensemble.

Figure 2. Estimate of virtual materials glass transitions and expansion coefficients through the plot of the logarithm of the cell specific volume vs. temperature.

Figure 3. Resulting hydrostatic pressure vs. logarithm of the cell specific volume during volume contractions through NVT ensemble at $350 \mathrm{~K}$.

Figure 4. Cells 1 and 2 bulk modulus vs. temperature. 
1

2

3

4

5

6

7

8

9

10

11

12

13

14

15

16

17

18

19

20

21

22

23

24

25

26

27

28

29

30

31

32

33

34

35

36

37

38

39

40

41

42

43

44

45

46

47

48

49

50

51

52

53

54

55

56

57

58

59

60
Figure 5. Normalized bonded energy of cell vs. specific volume resulting from NVT simulations at constant temperatures $350 \mathrm{~K}, 250 \mathrm{~K}$ and $150 \mathrm{~K}$.

Figure 6. Van der Waals energy per unit mass of the cell vs. specific volume resulting from NVT simulations at constant temperatures $350 \mathrm{~K}, 250 \mathrm{~K}$ and $150 \mathrm{~K}$.

Figure 7. Superposition of the data plotted in Figure 6 showing the independence on temperature of the changes of the Van der Waals energy vs. volume changes.

Figure 8. Fit of the cell Van der Waals energy variations with volume by a 9-6 Lennard-Jones potential: a)cell 1, b)cell 2 .

Figure 9. Comparison of the Van der Waals energy contribution to the bulk modulus with the material bulk modulus: a)cell 1, b)cell 2 . 


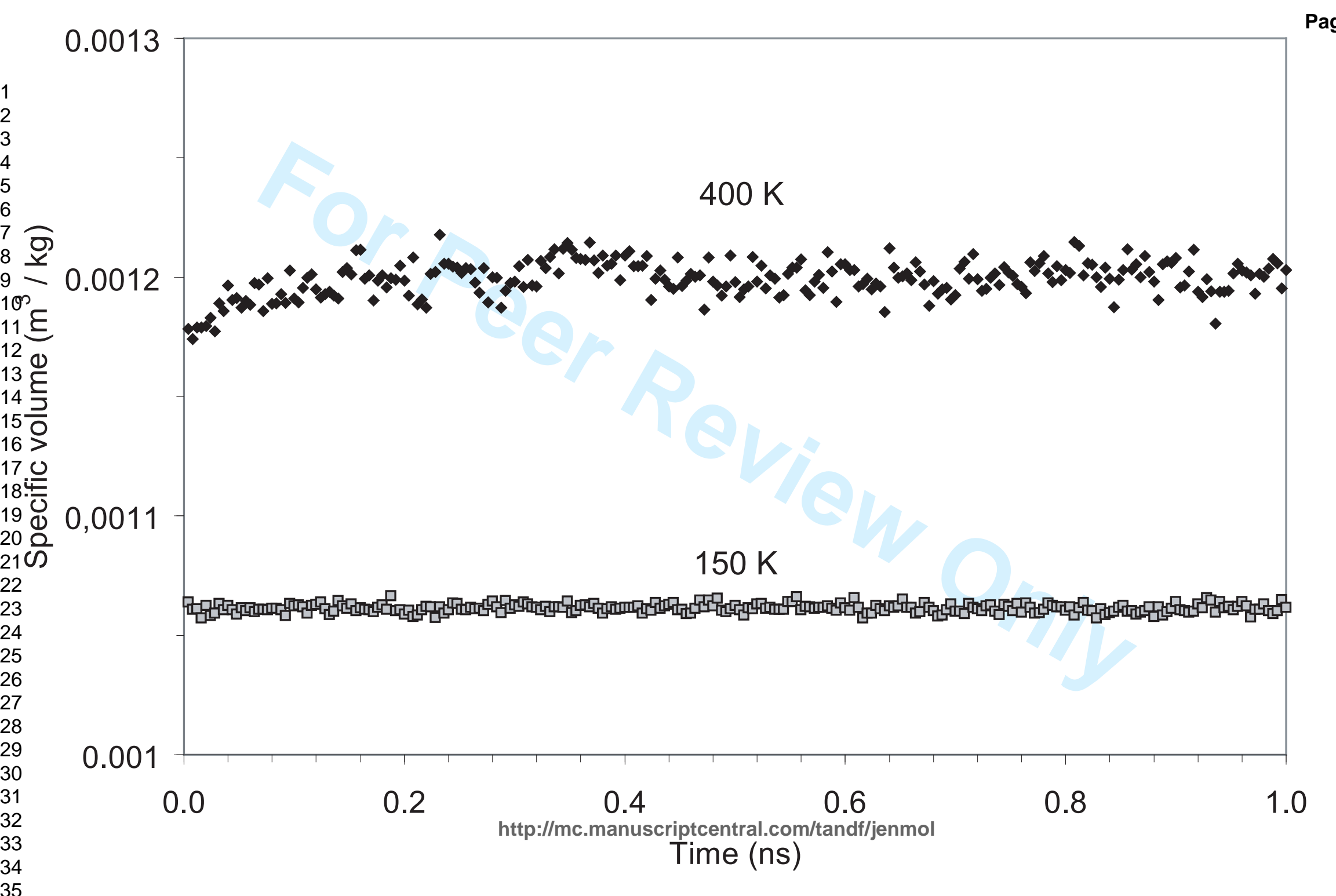

Page 10 of 18 


\section{Page 11 of 18}

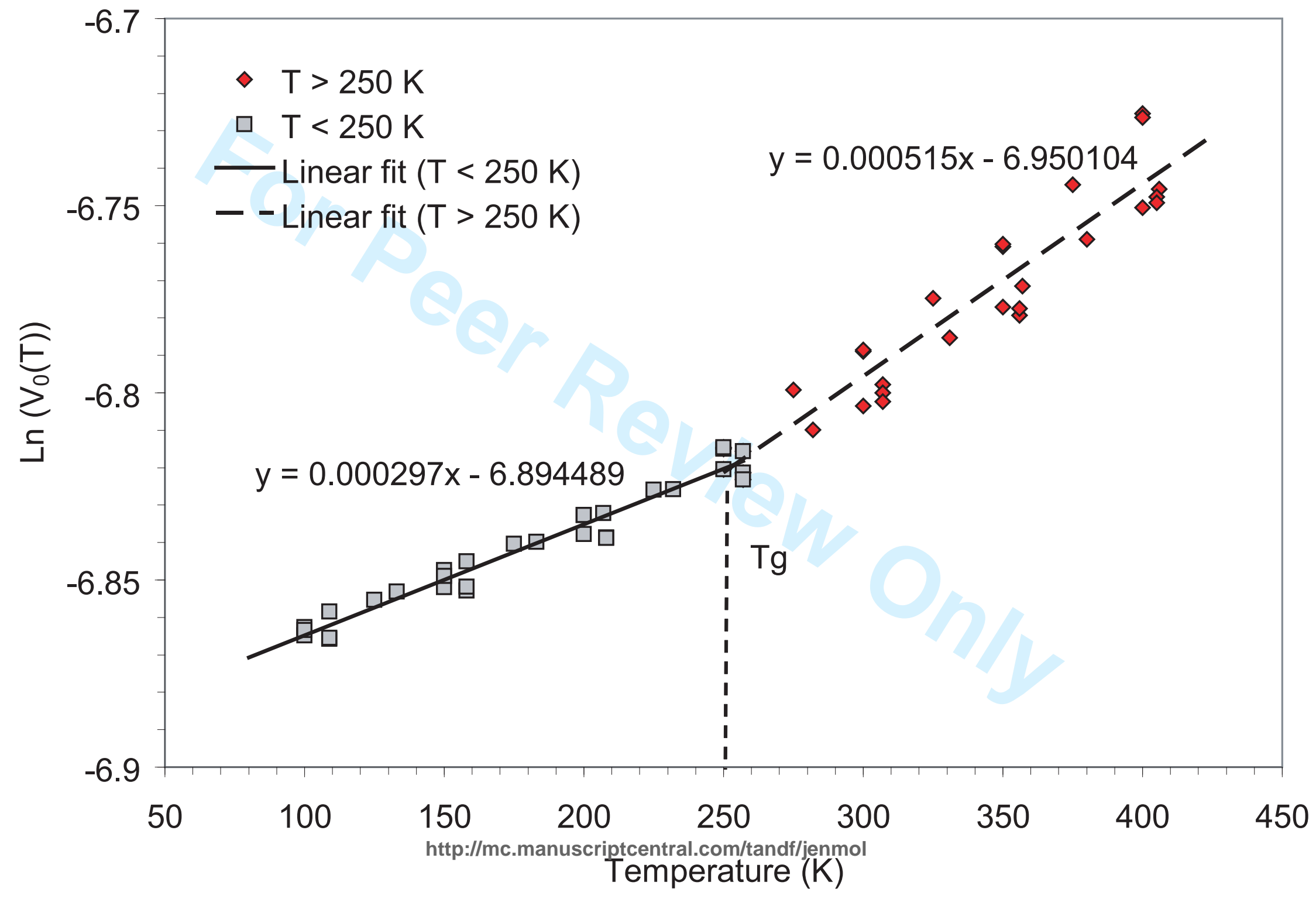




\section{Page 12 of 18}

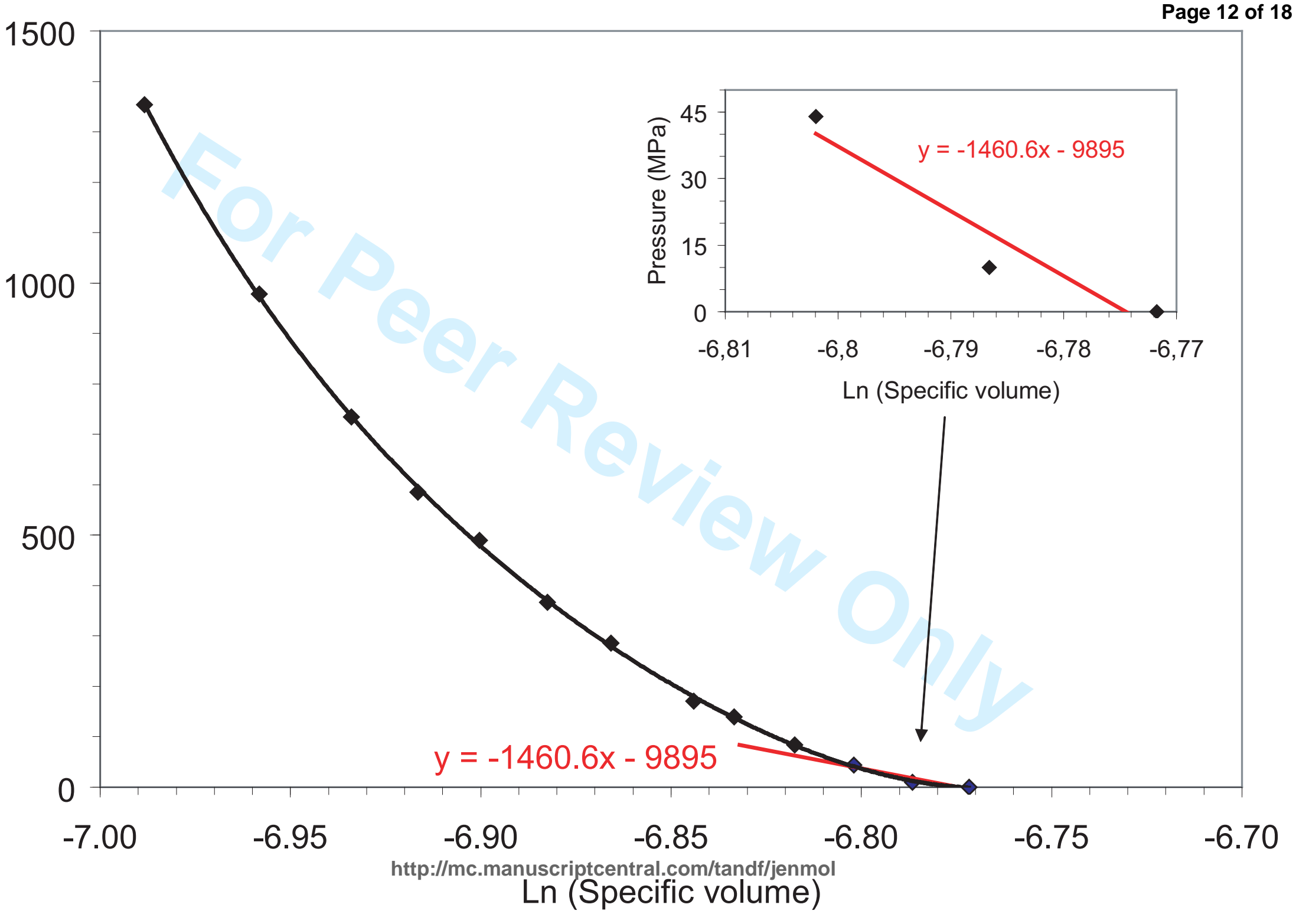



b)
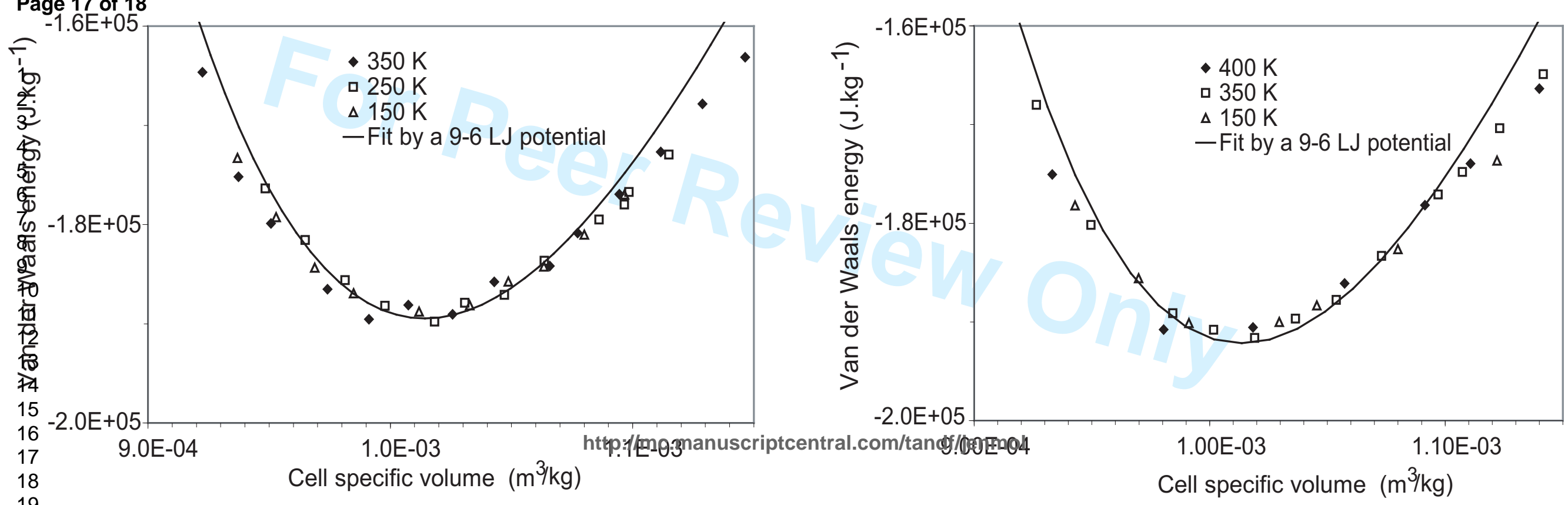
a) $\begin{array}{ll}\text { b) } 10000 & \text { Page } 18 \text { of } 18\end{array}$

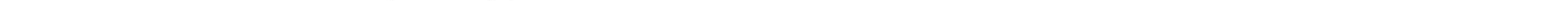

